\title{
SMTHSONIAN
}

पBRARIES 

Fabert:Voyaje au pays aes Trarzas. ( $B S G$ ). 
cessé de plaire, non sans motifs, au gouvernement du Sénégal, avait pour lui les deux grandes tribus nobles et guerrières des Oulad-Daman et des Oulad-Aïd; Ahmet avait. une portion au moins équivalente des guerriers Trarzas; la dernière fraction observail la neutralité. Mais Ahmet avait en outre les sympathies non déguisées du gouverneur du Sénégal; de plus le concours effectif du vaillant chef de la province noire du Oualo, Yamar, lui était acquis à cause de leur parenté. C'était d'ailleurs chez Yamar, sur la rive gauche du Sénégal ${ }^{1}$ qu'Ahmet-Saloûm s'était réfugié et qu'il faisait les préparatifs d'une guerre à outrance.

Les dangers de cette situation firent que le gouverneur du Sénégal me défendit d'abord de traverser le pays des Trarzas. Je songeai alors à passer par celui des Braknas, où j'avais résidé à trois reprises et oủ je compte de nombreux amis. Mais les Braknas étaient à ce moment fort mécontents d'avoir vu supprimer leur coutume annuelle, et le fils aîné du roi Sidi-Ely, Ahmedou, venu à Podor où je m'étais rendu et où je l'avais fait appeler, prétexta que la crue des eaux rendait le passage impraticable dans le Chamama et partit sans m'emmener avec lui.

Comme je manifestais la ferme volonté de ne me laisser arrêter par aucun obstacle et de gagner à quelquę prix que ce fùt le Sahara, le gouverneur du Sénégal, revenant sur sa première décision, m'engagea à passer par le pays des Trarzas, et il autorisa Yamar à me convoyer jusqu'au camp de guerre d'Ahmet-Saloûm, qui venait de passer le fleuve et qui se trouvait à la pointe nord du lac Cayar. Aussi,

1. On sait que toute la rive gauche du Sénégal est habitée par des noirs annexés ou protégés et que toute la rive droite, est occupée par Jes Naures indépendants. Nais en décembre 1891 des traités de protectorat ont été signaés avec plusieurs de ces derniers.

2. La " coutume " instituée par Faidherbe se paye en pièces de guinée (cotonnarle bleue). Elle représente la valeur de l'impôt que prélèveraient, à la sortie du pays, les rois maures, sur la gomme portée aus escales françaises. 
après de longs retards, je partais le 5 septembre de Dagana avec ma mission, qui se composait: $1^{\circ} \mathrm{de} \mathrm{M}$. Georges Descemèt, écrivain à la direction de l'intérieur, et fils de l'honorable ancien président du conseil général de la colonie; $2^{\circ}$ de Mamadou-bou-el-Moghdad, interprète et rédacteur d'arabe du gouvernement, fils du célèbre marabout qui, en 1860, partit de Saint-Louis comme pèlerin et gagna Mogador en traversant tout le Sahara. C'est avec ce personnel restreint, mais assurément choisi, que je devais accomplir un voyage auquel les circonstances m'ont empêché de donner tout le développement que j'eusse souhaité, et qui, par suite des troubles qui régnaient partout, n'était pas exempt de difficultés.

Nous traversâmes en une petite journée le pays de Chamama ${ }^{1}$ pour aller coucher au village ouolof de Kheo, établi en pays maure ${ }^{2}$, et oú nous passâmes une nuit cruelle, en proie à des nuées de moustiques, tandis que des rafales de pluie éteignaient les feux qui nous protégeaient seuls du fléau. A Kheo nous refìmes le chargement de nos chameaux, et une deuxième et forte étape, à travers les grasses prairies de la rive occidentale du lac Cayar, nous mena jusqu'à Berrek, dans un camp d'harratines (affranchis) qui tenait pour Amar-Saloùm. Aussi Yamar s'empara-t-il du chef du camp et le garda-t-il à vue toute la nuit, en le menaçant de mort au cas où les Oulad-Daman, avertis de notre présence, viendraient attaquer la mission.

Le 7, dans la matinée, nous rejoignions à N'Draya le camp de guerre du prétendant. Ce camp, de forme circulaire, était muni de retranchements bien compris. J'y fis connaissance avec Ahmet-Saloûm, un fort beau garçon de 24 à 25 ans, très intelligent et paraissant doué d'énergie.

1. Chamama, en maure, c'est la bande de terre soumise aux inondations annuelles du fleuve. Oualo, en ouolof, a la même signification.

2. Kheo n'est pas le seul village noir qui se trouve sur la rive droite du Sénégal.

SOC. DE GÉOGR. - $3^{\circ}$ TRIMESTRE 1892.

XIII. -25 
Ahmet, qui sentait tout le prix de l'appui des Français, me reçut courtoisement, mais lorsque je lui demandai de m'expédier le lendemain, arec une escorte, dans le nord du pays, il me répondit qu'il ne le pouvait faire avant quatre ou cinq jours, alléguant que le sort de la guerre civile serait fixé dans ce délai, et qu'en tout état de cause il ne pouvait actuellement point détacher de son armée un groupe quelconque de guerrier's. Je dus m'incliner devant la justesse de ces arguments, mais, par cela même, le sort de la mission se trouvait dès lors étroitement lié à celui du jeune prince trarza.

Le lendemain 8 , nous fìmes une marche pour contourner le lac et, en même temps, les camps des Oulad-Daman et des Oulad-Aïd, dont on entendait l'ésonner le tam-tam de guerre, et nous faisions jonction dans un endroit boisé appelé Oum-Lahbal avec un contingent de 730 Braknas venus des bords du lac Aleg ${ }^{1}$, en cinq jours de marche, pour donner leur concours à Ahmet-Saloûm² ${ }^{2}$. Dès ce moment l'armée du prétendant était au complet. Elle se composait d'environ 1,300 Trarzas, à pied, à cheval ou montés sur des chameaux, des 730 Braknas, la plupart à pied; de 200 fantassins et de 60 cavaliers noirs appartenant à Yamar, et passés avant lui sur la rive droite du Sénégal.

Le 9, nous achevions le mouvement tournant. L'armée marchait en bon ordre; elle subissait évidemment l'ascendant de Yamar, habitué à faire colonne arec nos spahis et nos tirailleurs. Le coup d'œil était intéressant. Tandis que les éclaireurs battaient la campagne à notre droite, du côté de l'ennemi, les Ouolofs, formés en deux compagnies de

1. Le lac Aleg, découvert par Bourrel en 1860. Aleg veut dire lac dans la langue du pays. C'est donc un pléonasue qu'on éviterait en lésignant l'Alér des brakıas sous le nom de lac de bourrel.

2. Les Braknas avaient à se venger de leurs ennemis séculaires, les Oulad-Daman, et aussi d'Amar-Saloùm, qui avait divoreć avec Mladjouba, sa femme, fille ainée de Sidi-kly, leur roi. 
LE PAYS DES TRARZAS ET LE SABARA OCCIDENTAL. 379

100 hommes marchaient en bon ordre, à l'aile droite. Au centre les Trarzas. Les harratines s'avançaient de front sur deux lignes, en chantant un air guerrier d'un rythme doux et grave. A gauche les contingents braknas, dans un ordre pareil. Vers midi et demi, du haut d'une colline, nous dominions le lac Cayar. A nos pieds, à 600 mètres sur la droite, un camp immense se déployait, paisible; on y voyait circuler des femmes et des enfants. L'ennemi, évidemment, ne nous attendait pas encore, ou du moins pas de ce cốté.

Dans un conseil tenu la veille avec Yamar j'avais décidé que la mission garderait la neutralité, à moins que l'ennemi ne tirât sur elle. Les Oulad-Aïd ayant commencé le feu nous n'avions plus qu'à nous défendre. Cependant notre petite armée s'était alignée correctement entre les collines et le lac. Au signal donné par Yamar la cavalerie partit au galop, franchit à gué un marigot et, suivie de près par les fantassins, enleva le camp des Oulad-Aïd. Ceux-ci se replièrent sur les Oulad-Daman en disputant le terrain pied à pied. Mais la charge, menée avec furie, les refoula au nord-ouest. Dès le début de la bataille Amar-Saloûm, dont on eût attendu mieux, s'élait enfui avec trois cavaliers, jugeant tout perdu pour lui, et avait galopé au nord-est pour se réfugier chez le chekh Sidia, grand marabout, qui réside entre le pays des Braknas et celui des Trarzas ${ }^{1}$. A trois heures et demie tout était fini. Les 1,700 hommes de l'armée royale étaient en déroute et vers quatre heures on m’apportait les premiers blessés qu'il me fallut panser, et dont le stoïcisme nous inspira une véritable admiration. Chose rare dans ces contrées, près de 4,000 hommes s'étaient trouvés sous les armes à cette bataille de Sehoutelma, qui vit s'effondrer la royauté d'Amar-Saloûm. Les pertes de l'ennemi avaient été beaucoup plus considérables

1. Cheilh (chirh), comme je l'expliquais plus haut, se dit d'un chef militaire; chekh (cherh) se dit d'un chef religieux. 
que les nôtres, ce qui s'explique par la rapidité foudroyante de notre attaque ${ }^{1}$.

Après cinq jours passés au partage du butin, Ahmet tint sa parole et m'expédia avec un contingent de tributaires Arrouijat qui regagnaient leurs camps, situés au nordouest, dans le pays d'Harya.

Une longue marche nous mena, le 16, au puits de BouTelleoulakt, dans le pays d'Iguidi, couvert de collines orientées le plus souvent du nord-est au sud-est. C'est la région des gommiers et l'acacia verek était en fleurs et dégageait une odeur suave. Le sol élait semé des graminées que fait croître l'hivernage; mais à Bou-Telleoulakt nous quittions tout à fait la zone des mares pluviales et nous étions désormais sous le régime des puits, souvent très profonds, qui assurent la circulation dans le Sahara.

Le lendemain soir nous étions au puits de Tinchikil, dont l'eau à peu près propre, mais légèrement salée, nous parut excellente, car nous avions déjà fait connaissaı e avec la soif. Une nouvelle étape nous mena à N'Tmahadi, où une violente tornade nous inonda et nous glaça toute la nuit: c'était d'ailleurs, depuis le Sénégal, notre rente habituelle dès la chute du jour.

A partir de N'Tmahadi les gommiers se font rares; ils ne sont plus les seuls arbres que l'on rencontre, ils sont mélangés aux tala et aux taïchit ${ }^{2}$. Presque aussitôt la végétation arborescente cesse; une terre désolée, dont la perspective est indiquée seulement par des genêts hauts de 4 à 5 mètres et par des touffes d'alfa ou de morokouba ${ }^{3}$; çà et

1. Yoici des chiffres que je crois exacts : armée d'Amar-Saloùm, 50 morts et 150 blessés; armée d'Ahmet, 5 morts et 70 blessés.

Plusicurs fenmes des Oulad-Aïd furent tuées dans le camp ct 4 furent blessées, auxquelles je donnai des soins.

2. Le lula est un arbre épineux, le taïchit est "l'arbre sans feuilles》 qu'on rencontre un peu partout dins le Soudan. Il a, en guise de feuilles, de longues ćpines verdàtres, peu acérées. C'est le soump des Ouolofs.

3. Le moroliouba est un panicus. Sa graine donne une farine assez bonne. 
LE PAYS DES TRARZAS ET LE SAHARA OCCIDENTAL. 381 là aussi l'affreux initi (le khakham des Onolofs), une herbe qui couvre le passant de petites boules aux mille pointes aiguës et adhérentes. C'est le pays d'Harya, ou " terre du néant $\triangleright$. Toutefois les pluies annuelles y font pousser pendant quelques semaines des petites fleurettes dont les chèvres et les moutons font leur régal.

Il existe quelques mares dans le pays d'Harya, notamment celles de Touidermi et d'Abouizir. Ces dernières correspondraient-elles au puits appelé Ouirir sur les cartes? Je ne le crois pas, bien que leurs positions ne diffèrent pas beau. coup; d'ailleurs, je ne connais pas de puits à Abouizir. Toute cette contrée a un aspect morne qui serre le cœur.

Le 19 septembre, dans l'après-midi, nous arrivions à un premier camp d'Arrouijat. Il était temps; mon chameau, très fatigué, s'était abattu et j'étais très souffrant de plaies que le soleil m'avait faites aux jambes (je portais le seroual, caleçon court des Maures), plaies que le contact des blessés de Sehoutelma avait rendues malignes et que la privation de l'eau qu'il m'eût fallu pour les panser avait, depuis lors, cruellement aggravées. Ma monture ne pouvant plus me porter, j’avais dû finir l'étape à pied, sous un ciel de feu. A 2 kilomètres du camp des Arrouijat il m'avait été impossible de continuer, et l'on m'avait fait à la hâte un abri en brisant au-dessus de ma tête les branches d'un genêt. Pour comble de malheur, ceux de nos gens qui portaient notre maigre provision d'eau sur leur chameau avaient pris les devants. J'étais donc étendu, dévoré par une fièvre intense, dans l'altente du secours. Des bergers qui passaient et qui me virent dans ce triste état furent sans doute les auteurs du bruit qui se répandit chez les Trarzas que j'étais mort de soif.

Au camp d'Argoub e reçus la meilleure hospitalité et le 22 la mission repartail pour le camp d'Abouizir; je m'étais fait hisser sur un chameau frais, mais de nouvelles souffrances me retinrent jusqu'au 26 à Abouizir. Les Arrouijat 
de ce camp furent pour moi d'une bonté parfaite; j'ai eu souvent l'occasion de voir que les Maures ne sont pas inhumains comme on se plaît à le croire et les mauvaises gens ne sont assurément point, chez eux, la majorité.

Du 'Trarza j'avais à me rendre au camp du chekh SadiBou ${ }^{1}$, illustre marabout saharien qui possède une réputation de sainteté de l'Ouad-Noun à la Gambie et du littoral de l'Océan jusqu'au Soudan. J'avais fait sa connaissance à Saint-Louis pendant un de ses rares séjours dans la colonie; je le savais doué d'un esprit ouvert et avisé, et de plus très partisan de l'influence française. Il m'avait d'ailleurs autrefois invité à l'aller voir, et sachant - les nouvelles circulent au désert avec une extraordinaire rapidité - que je me trouvais chez les Arrouijat, redoutant pour la mission l'insécurité actuelle du pays, il envoyait au devant de nous son frère, le chekh Baba. Ce marabout fit une marche forcée pour me rejoindre à Abouizir et m'exposa que si je me confiais entièrement à lui je pourrais traverser sans accident les parages oú rôdaient les Oulad-Delim et oủ des Aleb dissidents, partisans d'Amar-Saloûm, battaient également la campagne. Mais pour bénéficier de la protection due à la haute influence du chekh Sadi-Bou et à la sienne propre, il fallait que je remisse notre sort entre ses mains. Baba n'admettait pas que je conservasse l'escorte armée que les Arrouijat étaient prêts à me fournir. J'optai pour la protection des marabouts.

Baba avait amené avec lui plusieurs talibés ${ }^{2}$. Il amenait aussi un personnage dont la vie depuis plus de six ans confinait à la légende, un caporal d'infanterie de marine qui avait déserté en 1885, pour une peccadille, et qui avait adopté le costume, la religion et l'existence des Maures. Ce ne fut point sans curiosité que je vis cet homme dont on

1. Son vrai nom, celui qu'on lui donne au désert, est Saadh Boû.

9. Talibé, élève, disciple religieux. Ce mot a cours parmi les noirs musulmans. Le mot en arabe maure est telmirli. 
LE PAYS DES TRARZAS ET LE SAHARA OCGIDENTAL. 383

a tant parlé dans la colonie du Sénégal. Marthe, c'est son nom, s'est totalement identifié avec ses hôtes. Il se confondrait absolument avec eux si ce n'était sa barbe blonde, insolite chez les peuples du Sahara. Marthe, connu chez les Maures sous le nom de Mohammed-Abdallaye, me parut réellement intéressant et j'estime qu'il pourrait, comme il le désire du reste, rendre aux Français de grands services dans ces pays si difficiles à aborder. Désormais pendant deux mois cet homme ne devait plus me quitter, et je ne puis que le remercier du dévouement dont il a fait preuve.

Le 26 septembre nous partions d'Abouizir. Jusque-là nous avions marché, de N'Draya à Tinchikil au nord-ouest; de Tinchikil à Argoub et Abouizir au nord. Notre route sera désormais au nord-est jusqu'à Tenyera, oủ nous rencontrerons l'un des camps du chekh Sadi-Bou et le grand marabout lui-même.

Une forte marche nous conduit à Ouber-Echgarra, où nous recevons une hospitalité médiocre dans un camp de marabouts Id-Jacoub. Dans cette marche nous avions été torturés par la soif, car le puits de N’Tfachit, situé à la halte et où nous comptions nous désaltérer, venait de s'ébouler au moment de notre passage. Le sol recommençait à être accidenté et légèrement boisé. Les collines sont de sable rouge. Les arbres sont épineux, sauf quelques adriss, qui ont l'apparence de merisiers trapus et qui fournissent une résine purgative. Les graminées sont disposées par touffes; on rencontre aussi de grandes quantités d'alfa. D’Ouber-Echgarra nous gagnons le beau puits de N'Zamadi, après avoir fait halte dans un camp de marabouts Midelich. Près de N'Zamadi nous couchâmes dans un camp de marabouts Tagounantes, où vinrent rôder, pour nous espionner, une douzaine d'Aleb dissidents. Dans la nuit ces voleurs nous soustrayaient un lot de pièces de guinée et disparaissaient sans bruit. A l'étape du lendemain nous devions nous retrouver. Effectivement, le 29 septembre, prévenus dela 
probabilité d'une attaque, nous chargions nos armes, mais sur notre caravane, composée d'une dizaine de personnes, nous n'étions que quatre portant des fusils. On n'ignore pas que les marabouts, sauf dans certaines tribus guerrières, comıne les Oulad-Bou-Seba, sont neutralisés et ne portent jamais que des bâtons.

Nous étions en route depuis deux heures lorsque brusquement, avec unc habileté et une rapidité qui tiennent du prodige, un mechbour de douze guerriers Aleb nous enveloppait, et sans l'intervention de Baba le combat eût été inévitable. Il convient de noter que c'est ce même chekh Baba qui, sur l'ordre de son frère Sadi-Bou, sauva Soleillet, en 1879, des mains des Oulad-Delim. La place me manque pour relater ici l'enquête que la mission a faite et près des témoins oculaires de ce mémorable incident. Tout ce que j'en puis dire c'est que si la vie du célèbre voyageur a couru les plus grands risques, il est tout à fait inexact qu'il ait été pillé. Ses ballots de marchandises, intacts, furent amenés avec lui chez le chekh Sadi-Bou, et il fit don à ce marabout, en témoignage de gratitude, de tout ce qu'il possédait.

Après des pourparlers qui eurent lieu sans interrompre notre marche, et pendant lesquels nos interlocuteurs tenaient, comme nous, le doigt sur la détente de leurs fusils, la paix fut faite, gràce au chekh Baba et aussi à notre ferme contenance. Nous donnåmes un peu de tabac et de poudre et nous rîmes s'éloigner - momentanément - ces compagnons importuns. Ils devaient revenir pendant trois ou quatre semaines avec d'autres Aleb, demander nolre tête au chekh Sadi-Bou. Enfin vers midi nous étions chez celui-ci, où une réception cordiale, mais non pas luxueuse ni bien confortable nous attendait. Nous étions arrivés dans le pays de Tenyera, c'est-à-dire dans cette partie vague du sud saharien où circulent des camps de marabouts et des caravanes que rançonnent sans scrupule les Oulad-Delim. 
LE PAYS DES TRARZAS ET LE SAHARA OCGIDENTAL. 385

Le chekh Sadi-Bou est un hoinme de 48 ans, assez basané. Quand il parle à des étrangers ou quand il voyage, il a la figure presque aussi voilée que celle des Touaregs; d'ailleurs il est originaire du Haodh, où son frère aîné est établi dans la ville d'Oualata. Son cadet, le chekh Nm'El-Haïnin s'est fixé dans l'Ouad-Saghié, au sud de l'Ouad-Noun. Il est, m'a-t-on dit, en excellents termes avec l'empereur du Maroc, qui lui envoie souvent des cadeaux, et il a pour mission spéciale de s'opposer aux empiètements des Anglais sur les territoires du cap Juby. Il est făcheux que nous ignorions presque entièrement, par notre faute, ce qui se passe dans cette partie du Sahara, qui nous intéresse cependant d'une manière directe.

Contrairement a la coutume des Maures, qui sont presque toujours monogames, le chekh Sadi-Bou a quatre femmes, et il a établi dans une tente, comme le lui permet la loi musulmane, une de ses captives dont il a plusieurs enfants. Sa nombreuse progéniture se compose de sept fils et d'une dizaine de filles, qu'il marie modestement à des talibés de sa suite ou à des marabouts éloignés.

D’une intelligence à la fois aiguisée et pondérée, fort lettré, ferré sur les textes sacrés comme sur les écrits profanes, ce grand marabout exerce un ascendant inouï sur tous ceux qui l'entourent. Exempt de fanatisme, rendant pleine justice à la droiture des Français, il est pour nous le plus admirable agent d'influence qui se puisse voir dans le Sahara occidental. Il serait bien à souhaiter que la France, autant pour reconnaître ses services que pour développer sa puissance morale, dont elle bénéficie largement, payât à ce chekh éminent les frais d'un voyage à la Mecque, comme elle le fit en 1860 à Bou-el-Moghdad. Ce seraient quelques milliers de francs placés à gros intérêt.

La mission était à l'abri chez le chekh, mais elle y était cernée. Les Oulad-Lab, qui sont une fraction des OuladDelim, les Aleb et d'autres coureurs du désert nous 
gardaient en quelque sorte à vue dans le camp du marabout et il nous était impossible de nous écarter des tentes dc Tenyera. Force nous fut d'atlendre là que l'apaisement se fit. Je profitai de ce séjour prolongé pour étudier les mœurs des musulmans de cette sorte de monastère. J'avoue que si elles ne sont pas toujours exemptes d'hypocrisie, elles sont en général douces et recommandables. Cependant les captifs et les captives y sont non pas maltraités, mais nourris d'une façon bien insuffisante. Cela tient peut-être à la misère profonde que le manque d'eau, depuis deux ans, a provoquée dans ces contrées sahariennes. Pendant les quarante-sept jours que nous passâmes chez le chekh Sadi-Bou, soit dans le pays de Tenyera, soit dans celui d'Harich, il n'est tombé que deux averses, et c'était la saison des pluies!

De Tenyera nous montâmes au nord, à Harich, où nous fùmes le 9 octobre. Je n'étais plus qu'à trois bonnes journées de l'Adrar et j'envoyai à Ahmed-ould-Soueyd-Ahmed-ould Aïda ${ }^{1}$, cheikh des Yahia-ben-Osman, une lettre amicale, par deux notables talibés. La réponse m'arriva quelque temps après. Elle était des plus cordiales et contenait une acceptation formelle du protectorat de la France. Ahmed, que j'avais connu en 1890 chez les Maures Braknas, au camp du roi Sidi-Ely, son oncle, me donnait des gages de sérieuse amitié, mais prêt à repousser une invasion des Maures l'Dowiches, il ajournait à plus tard ma réception dans son pays, craignant de m'exposer alors à des dangers inévitables; mais le but politique du voyage était atteint.

Le calme s'était rétabli relativement dans la région; ne pouvant prolonger davantage mon séjour, muni d’ailleurs de l'excellente réponse d'Ahmed-ould-Aïda, je pris le parti de gagner l'Atlantique. Le 16 novembre, à 10 heures du soir,

1. Soueyd, diminutif : petit Sidi. Terme familier qui est remplacé dans les actes publics par le nom de Sidi. 
pourvue de chèvres et de moutons que poussait un talibé à pied et gratifiée d'une forte guerba de dattes de l'Adrar, la mission faisait ses adieux au chekh Sadi-Bou. Une première étape, de nuit, nous mena dans un camp de marabouts Ehel-Mouhbarek; une deuxième, de 70 kilomètres, nous faisait coucher à Teniafouil, où était passé avant nous le mulâtre Léopold Panet, en 1850. Le lendemain une étape de 80 kilomètres nous conduisait, à travers le pays d'Amoukrouz, qui monte beaucoup plus dans le nord que ne l'indiquent les cartes, - jusque dans le pays de Tafoualli, à Lemonek. Enfin, le lendemain 19, une autre marche forcée nous amenait sur le bord de l'océan et nous saluions avec joie les flots de l'Atlantique.

Depuis Harich le terrain est très accidenté. Le 16 nous avions coupé une série de belles vallées, larges et profondes, dont l'une est appelée le Grand-Terg (Targa-Kebira). Cette vallée, dont huit jours plus tard nous déterminâmes la naissance à Aghangelit, dans l'Afftouth, est la grande artère de communication de toutes ces contrées; elle va d'abord d'Aghangelit au nord-est, infléchit au nord au-dessous de Teniafouil, et tourne enfin à l'est pour aller mourir entre l'Adrar et le Tagant.

Entre le Tafoualli et l'Afftouth (c'est-à-dire la plaine qui borde partout l'océan), s'étend la longue bande nord-sud du pays de Tarad. Pour arriver au littoral même, notre caravane dut traverser les deux chotts de Leghrik, passage dangereux, étroit, où chacun mettant pied à terre conduit son chameau par la corde et marche soigneusement dans les traces de pas existantes, sous peine d'enlisement.

L'Amoukrouz est plein d'une espèce de caoutchoucs nains qui dégagent une odeur vineuse caractéristique; Vincent les a indiqués comme étant des euphorbes. Au nord de ce pays, se trouve un gisement de soufre. Dans le Tafoualli et le Tarad la végétation, clairsemée, se compose de tala qui atteint de 4 à 5 mètres de hauteur, et de tama- 
ris. C'est encore ce dernier qui se montre dans l'Afftouth, et nous avons dormi plus d'une fois abrités derrière ses touffes épaisses contre le vent glacial du nord-ouest. Cet arbrisseau ne garnit d'ailleurs que les plaines et le fond des vallées; dès que le terrain s'accidente, le caoutchouc nain reparaît. Comme dans l'Harya et l'Harich, les vipères roses, dont la morsure est mortelle, sont abondantes; elles se terrent sous les souches des caoutchoucs nains. Mais les énormes scorpions jaunes qui jonchent l'intérieur ne se voient pas sur le littoral.

J'étais rendu à Djéil (ou Toueil), voisin des puits de N'Dramcha et de Blaiouak. A Djéil l'eau est abondante, à une faible profondeur, comme sur toute la côte, et, chose bizarre, moins salée que dans l'intérieur du Sahara. Sur ce point nous pûmes voir l'épave du Montesquieu, que signalent les cartes marines. Quand à Andiil, ou le Vieux Portendik, porté sur les cartes, il est tolalement inconnu des Maures. L'einplacement du Nouveau Portendik, c'est à Marsa qu'cn le trouve, à une petite journée dans le sud.

Je m'y rendis le 20 novembre, après avoir admiré à Djeil la richesse inouïe des pêcheries qu'y ont établies les Ouladbou-Seba de la tribu des Min-Hannab, rameau détaché de la grande nation du nord-ouest du Sahara. A Marsa jẹ retrouvai, curieux souvenir, au bord de la superbe saline de Jor, les vieux canons du fort qu'un Français nommé Houlquije (d'après les gens du pays) occupait au xvir siècle. Houlquije parlait très purement l'arabe et composait même des vers en cette langue. Marsa devait être un emplacement commercial magnifique ${ }^{1}$. La mer n'y brise pas, garantie qu'elle est, par le banc d'Arguin, de la houle du nordouest et les tribus de marabouts qui campent dans le voisinage, et qui sont le plus souvent des Tendagha

1. Marsa en arabe signifie escale. C'est donc bien là réellement qu'était Portendik. C'est l'endroit que les cartes appellent Djioua ou le Noureau Portendil. 
LE PAYS DES TRARZAS ET LE SAHARA OCCIDENTAL. 389 (on prononce Tendrha) sont douces et commerçantes.

Voici quelles sont les étapes de la fin du voyage :

De Marsa à Toujounine, en passant par Mousserane, où l'eau douce abonde à un mètre dans le sol et où l'on ferait aisément une fertile oasis; par Boul-Erhouara (le « maître du gommier ») et par Nouakchot. Ces deux endroits sont munis de puits.

De Toujounine à Tivourvourt, (et non Tiourourt comme l'indiquent les cartes), en passant par le puits d'Aghangelit, commencement de la vallée du Grand-Erg;.par les puits de Giour, de Jaïrinié, d'Ahreiguib, qui prennent leur nom des collines voisines, que j'ai relevées. A Tivourvourt les datteries visitées il y a trente et un ans par le capitaine Vincent, lors de son beau voyage dans le Tiris, n'existent plus.

De Tivourvourt à Chéréha, en passant par les puits et la colline de Tamzagt, par les collines de Diabagh et de M'Bodiell et par les salines de Moulagcheb.

De Chéréha à Tinijat, en passant par les salines de Chéréha, de sob et par le joli lac de Bourrouk, de formation toute récente; par les puits de Tenheud; par les collines de Moulmaouje (la « maîtressé des vagues »), de M’Boudiane, de N'Fourdell, où nous vîmes de fort belles cultures créées par un marabout solitaire de la tribu méridionale des Koumleïleh, enfin par la colline de Sob.

De Tinijat à Ten-Hambar, par la colline et le puits de Bou-Hagera (la «maîtresse de la pierre )), par les collines de N'Mouktaria, de N'Hayma (la « petite autruche »), de M'Bsouth, de N'Tergueguit, de M'Béréou, de N'Dor et de N'Dorit, de Tinnamaten, de Nichfofoua, de Nebgati, de Tourja, qui donne son nom à une saline, de M'Betihé et de Takal (saline du même nom).

De Ten-Hambar à N'Diago, en traversant le marigot de N'Diadier ou des Maringouins, qui fait communiquer le grand coude du Sénégal avec la mer. Contrairement à l’itinéraire de retour de Vincent et à l'aller de Bou-el-Moghdad, 
nous passames à l'est du lac Taniahya. J'ai pu voir, après Ten-Hambar, la chaîne de collines tourner à angle droit et infléchir à l'est, formant ainsi la limite nord-ouest du pays de Chamama.

Le 29 nous couchions sur la plage de N'Diago, oủ nous nous reposions la journée du lendemain. Le soir nous entrions à Saint-Louis sur nos chameaux, après avoir altendu la nuit, car nous étions accoutrés d'une façon pitoyable. Les vêtements européens de M. Descemet étaient en loques; le costume de guinée maure de Bou-el-Moghidad et le mien ne valaient guère mieux. Il n'est pas jusqu'au drapeau tricolore qui avait fait avec nous cette courte mais rude campagne qui ne fût transformé en haillon.

\section{NOTICE COMPI_ÉNENTAIRE}

On ne saurait, attribuer sur une carte, sous peine de commettre une erreur, un emplacement précis aux tribus maures du Trarza ni à celles qui virent au-dessus de ce pays, dans le Sahara proprement dit, mais on peut dire d'une façon générale que les Guebelés habitent le sud, ainsi que cette dénomination l'indique, et qu'ils se tiennent aussi près que possible de la région du fleuve. Seuls les Oulad Daman peuvent être regardés comme ayant un territoire leur appartenant en propre; c'est celui qui s'étend entre le lac Cayar à l'ouest, le Chamama au sud, à l'est le Brakna, et au nord les pays mal définis qui vont de Tenyera a l'extrémité de la chaîne relevée par Bourrel, et au Tessageurt. Les Oulad Bou Seba, de la famille des Nin Hannah, étant des pêcheurs, parcourent exclusivement le littoral de la mer, de N'Diago à Achéil. En dehors de cetle classification les tribus se déplacent et entremêlent leurs camps sans autre riggle que leur fantaisie, leurs habitudes et les nécessités de l'eau et des pâturages. 
LE PAYS DES TRARZAS ET LE SAHARA OCGIDENTAL. 391

Voici la nomenclature complète des tribus de guerriers et des tribus de marabouts du Trarza, par ordre d'importance politique ou religieuse :

TRIBUS DE GUERRIERS

1. Oulad Ahmed ben Daman

2. Oulad Daman............

3. El Haeballah...........

4. El Agmoutar...........

5. Oulad el Bolya..........

6. Oulad Beniouk (Azounas).

7. Takhridiente...........

8. Oulad Akchar...........

9. Oulad bou Seba...........

10. Oulad Bohali............

11. L'Mradine.

12. Aleb

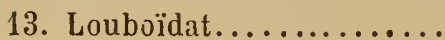

14. Arhalla...............

15. Oulad Aïd.............

16. Zombotti,.............

17. Oulad Rgueïk...........
Ne payent aucun tribut idem............

idem.............

idem..............

idem...............

idem..............

idem..............

idem...............

idem..............

idem..............

Paycnt tribut au cheikh des Trarzas..........

idem..............

idem..............

Payent une redevance à d'autres tribus...... idem............... idem............... idem..............
Origine arabe. idem.

idem.

idem.

idem.

idem.

idem.

idem.

idem.

idem.

idem.

idem.

idem.

Mélangée.

idem.

idem.

idem.

\section{ZENAGAS (origine berbère pure ou mélangèe de sang arabe)}

1. Arrouijat.

2. L'Mragui.

3. Oulad-abd-el-Ouahet.

4. Oulad Embarek.

5. Irombatten.

6. Degjmolla.

7. Lehempch.

8. Souyeilat.

9. Akfollaten.

10. Bassyne.

11. Soubak.

12. Sbehat.

13. Souhait.

14. Ideyrik.

15. Dragla.

16. Oulad el Vari.

1i. Dabagren.
Quelques-unes de ces tribus sont assujetties par fraction à des peuples maures autres que les Trarzas (Braknas, Idowiches, etc.).

Toutes ces tribus parlent la langue arabe hassania.

Quclques-unes se subdivisent en plusieurs brauches. 


\section{TRIBUS DE MARABOUTS}

1. Oulad Deymon.

2. Tendagha.

3. Id Jacoub.

4. Barekallah.

5. Tachidbit.

b. Koumleïleh.

7. N'Tabo.

8. Finel Rays.

9. Ehel Abieï.

10. Idab Lassen.

11. Taguenit.

12. Idaouali.

13. Midelich.

14. Tadjakante.

15. Tagounante.

16. Oulad Abieri.

17. Dehouel Hadj. (Darmankours).

Toutes ces tribus de marabouts parlent l'arabe. Quelques-unes, surtout la première, sont composées de iettrés et jouissent d'une grande autorité morale. Chez toutes le berber est parlé assez fréquemıent.

Aucune tribu de marabouts ne paye d'impòt aux gucrriers.

Cetle dernière tribu est très indépendante.

Chez les Trarzas comme chez tous les Maures, un grand nombre de tribus ont leurs camps d'harratines ou affranchis, qui prennent généralement leur nom.

Le Gerant responsable,

CiI. Maunoir, Secrélairc général de la Commission centrale.

4536. - L.-Imprimeries rónies, B, rue Mignon, 2. - MaY et Motrgroz, direct. 



\section{TRIBUS DE MARABOUTS}

1. Oulad Deymon.

2. Tendagha.

3. Id Jacoub.

4. Barckallalı.

5. Tachidbit.

b. Koumleïlch.

7. N'Tabo.

S. F.hel Rilys.

9. Ehel Abicï.

10. Idab Latssen.

11. Taguenil.

19. Idaouali.

13. Midelich.

1.1. Tadjakantc.

15. Tagounante.

16. Oulad Abieri.

17. Dehouel Hadj. (Darmankours).
Toutes ces tribus de marabouts parlent l'arabe. Quelques-unes, surtout la prcmière, sont composées de lettrés et jouissent d'unc grande autorité morale. Chez toutes le berber est parlé assez fréquemuent.

Aucune tribu de marabouts ne paye d'impôt aux gucrricrs.

Cette dernière tribu est très indépendante.

Chez les Trarzas comme chez tous les Maures, un grand nombre de tribus ont leurs camps d'harratines ou affranchis, qui prennent généralement leur nom.

\section{Le Gerant responsable,}

Cir. Maunolr, Secrétaire grénéral de la Commission centrale.

1536. - L.-Imprimeries róunies, B, rue Mignon, 2. - MAY ot Motrknoz, direct. 


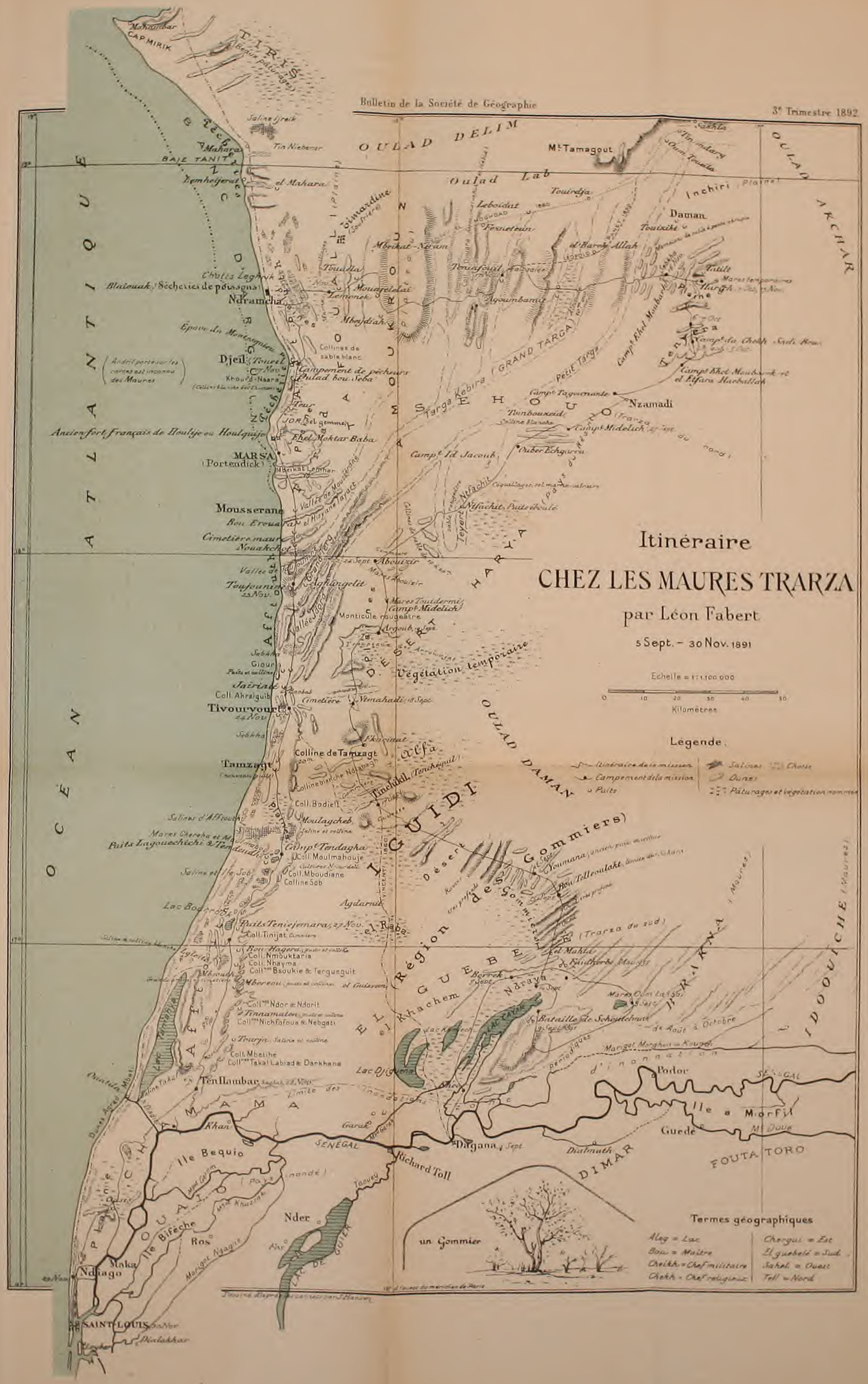






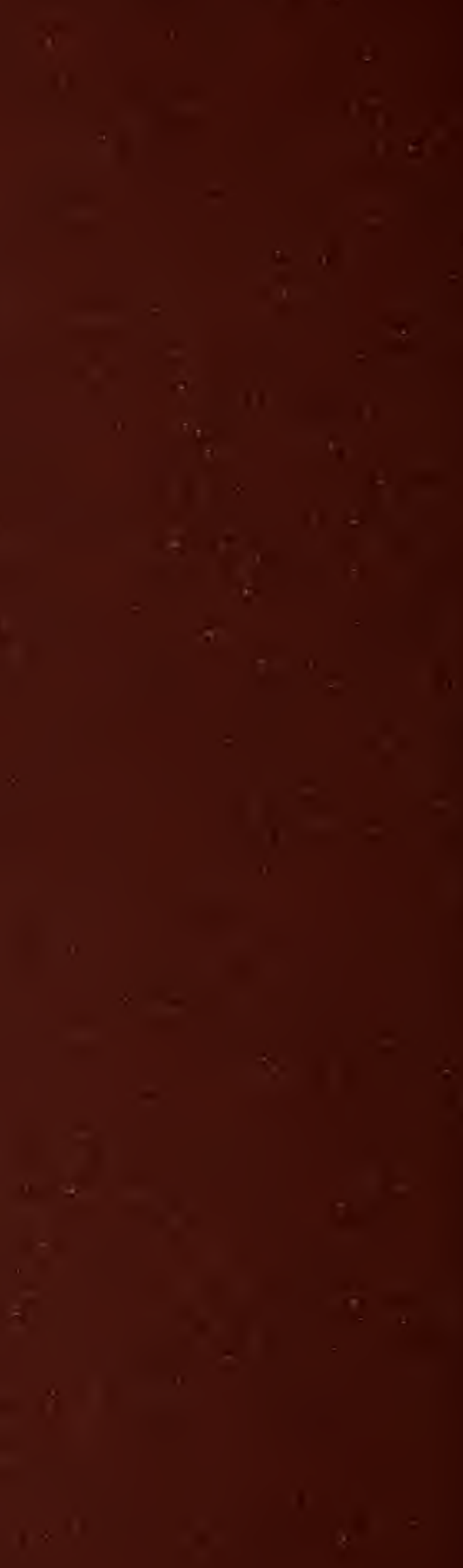




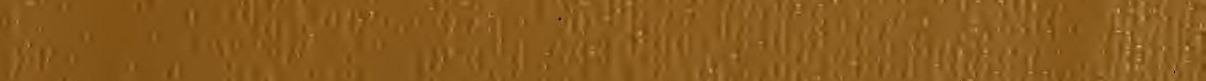

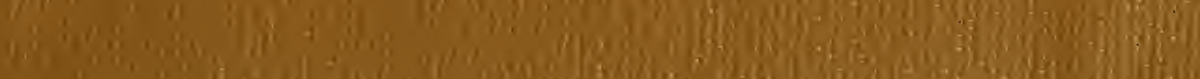

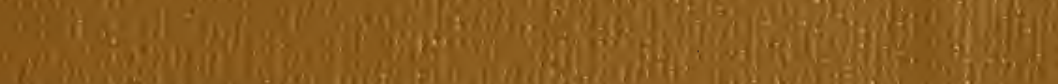

\section{-}




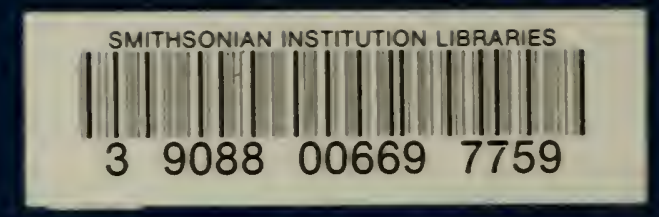

